\title{
Vision System for Subpixel Laser Stripe Profile Extraction with Real Time Operation
}

\author{
Ulises Martínez, Benjamin Valera, José Sánchez, and Victor García \\ Centro de Ciencias Aplicadas y Desarrollo Tecnológico, UNAM, Ciudad Universitaria, \\ 04510 D.F., México \\ metro@aleph.cinstrum.unam.mx
}

\begin{abstract}
Computer vision systems are playing an important role in 3D measurement for industrial applications. Real time image processing algorithms are useful in order to achieve reliability in feature extraction from the global environment starting from planar images. For example, in a structured light vision system is essential to extract the pattern that a laser source is shaping with the objects under inspection. In this sense, this work describes a single strip image extraction algorithm that could be used as an analysis tool in those structured light systems. The experimental setup is implemented using the following equipment: A PC equipped with a frame grabber, a high-resolution CCD camera, a laser stripe projector and specific software developed using Visual $\mathrm{C}++6$. The system accomplishes with real time operation and high subpixel accuracy.
\end{abstract}

\section{Introduction}

Recently, in our dimensional metrology laboratory, have been developed measurement techniques using computer vision with real time operation for industrial applications. Computer vision efforts are directed to reduce costs and operative time in the industrial field [1]. Additionally, measurement vision systems offer the great advantage of measuring without mechanical contact. However, vision systems still lack of high accuracy due to some restrictions in the image formation process. On the other hand, our aim to develop a measuring vision system is based on the utilization of auxiliary techniques that contributes with the high accuracy purpose. For example, the mechanical arrangement in the computer vision layout can be tested with higher accuracy standards, like coordinates measuring and form scan machines. Moreover, the accuracy in a measurement vision system is not only limited by the resolution of the acquisition devices; the planar image, highlighted by a structured light source, is improved and reduces some limitations in the image formation process. Also, the structured light contributes to simplification of the image management for feature extraction [2].

It is well known that computer vision techniques take advantage of subpixel feature extraction [6]. In particular, a structured light system needs to extract the strip image that is formed by the intersection of a laser stripe projector with the object under inspection. Then, relevant extracted features can be reconstructed in a 3D environment using the triangulation principle together with the camera and laser 
source calibration. Therefore, it is possible to perform 3D measurements using monocular vision [5].

This paper deals with the sub pixel laser stripe profile extraction in planar images incoming from a structured light system for metrological purposes. The aim is to create a real time operation system to be operated as a high accuracy measurement instrument. The relevance of our work is the development of a practical implementation, this is to apply a feature extraction algorithm to create a useful tool with industrial application. The organization of this paper is as follows: Section 2 presents the computer vision system principle for dimensional measurements. A review of an algorithm for laser stripe profile extraction is presented in section 3 . We give emphasis to in a closed form solution that meets accuracy and committed processing time. Section 4 describes hardware and software platform supporting our implementation. Section 5 presents some results of applying three-dimensional reconstruction to the feature extraction process. Finally, our work is summarized in section 6 .

\section{Measurement Principle Using Structured Light}

Our scheme for the dimensional measurement using computer vision is based on the well-known triangulation principle and the layout in Fig. 1(a). The hardware was selected in order to gain high accuracy at low cost. The laser stripe projector generates a sharp light pattern that intersects with the $3 \mathrm{D}$ object under inspection. Then the pattern is digitized with a CCD camera and a frame grabber attached to a computer. The resulting planar image contains simplified information about the object, useful for $3 \mathrm{D}$ reconstruction purposes. It is possible to demonstrate that a $3 \mathrm{D}$ reconstruction process of the 2D stripe pattern can be done by means of camera and laser plane calibration, as Fig. 1(b) shows.

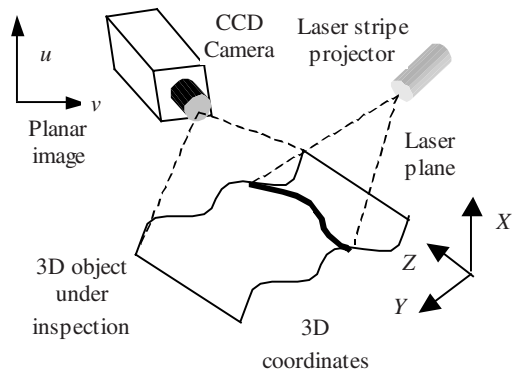

(a)

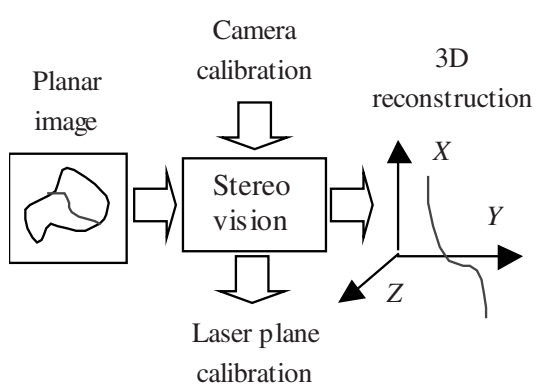

(b)

Fig. 1. Dimensional measurement using computer vision.

The layout of Fig. 1 involves the following main tasks:

1. Camera calibration. Implies to find the mathematical model that relates $3 \mathrm{D}$ global coordinates with its planar projection $[7,8]$. 
2. Laser plane calibration. It consists in finding the mathematical expression of the laser plane.

3. Stripe image processing. The main idea is to extract the spine of the stripe image with subpixel accuracy.

4. Reconstruction. This is the aim of the measurement instrument and takes as inputs the calibration process and the stripe image to generate 3D positions.

In this paper we focus mainly in the stripe image processing stage, due to its importance in the measurement process and its relevance in the automation of the procedure.

\section{Stripe Image Processing}

The aim of the algorithm described in this section is to extract the spine of the stripe image accomplishing with subpixel accuracy. The physical situation presented in Fig. 1 imposes planar stripe images, which pattern approximates a Gaussian distribution. Then, the feature extraction process provides a reduced set of descriptor parameters and also contributes with the improvement in the reconstruction. The algorithm is divided in two main stages: Image simplification in order to isolate the stripe profile and subpixel feature extraction in order to obtain accurately the set of parameters conforming the spine.

\subsection{Image Simplification}

The Fig. 2 shows typical planar images generated by the proposed layout in Fig 1 . The Fig. 2(a) shows the scene with no light from the laser stripe projector when it is turned off. In the Fig. 2(b), the laser plane intersects the scene when the projector is turned on. The Fig. 3(c) shows the absolute value of the difference between figures 2(a) and 2(b), isolating in this way the profile of the object under inspection. The laser projector is turned on and off by simple electronics and control software.

\subsection{Subpixel Feature Extraction}

In this stage [4], the image in Fig. 2(c) $I(u, v)$ is processed by establishing initial conditions for a center of the stripe $\mathbf{p}_{i}\left(p_{x i}, p_{v i}\right)$ together with a line width $w_{i}$ and a line direction $\mathbf{d}_{i}\left(d_{x i}, d_{y i}\right)$. The initial center of the stripe is obtained using a scan procedure from top to bottom and left to right in image coordinates. The extracted information conforms a line element $\mathbf{e}_{i}\left(\mathbf{p}_{i}, w_{i}, \mathbf{d}_{i}\right)$, as shown in fig 3(a). Then, starting from the initial conditions, the subsequent line elements are generated in the following way. The next line element $\mathbf{e}_{i}\left(\mathbf{p}_{i}, w_{i}, \mathbf{d}_{i}\right)$ is estimated by forming a circular pattern $t(\mathbf{p}, r)$ with center in $\mathbf{p}_{i}$ and radius $r_{i}=0.6 w_{i}$. The pattern is used as reference trajectory in finding the borders of the stripe $\mathbf{b}_{1} \mathbf{b}_{2}$, as shown in Fig. 3(b). The position, direction and width of the next element line are given by 


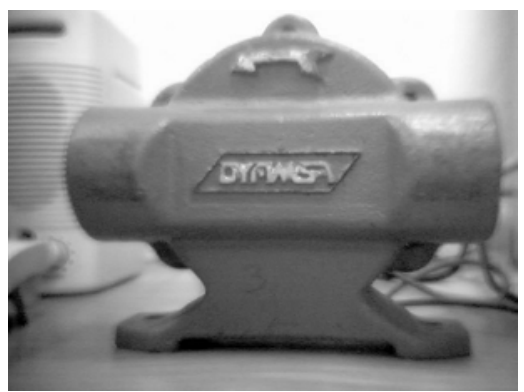

(a)

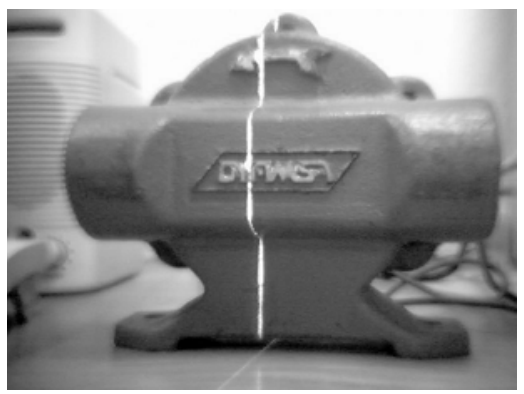

(b)

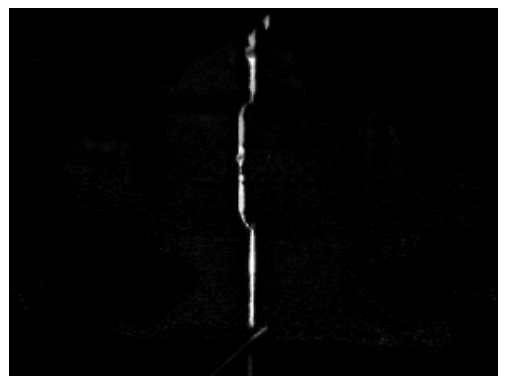

(c)

Fig. 2. Planar projections of the measurement system by vision

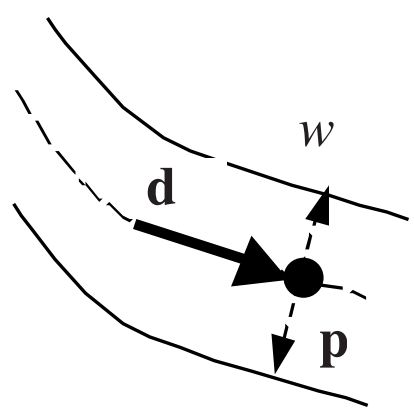

(a)

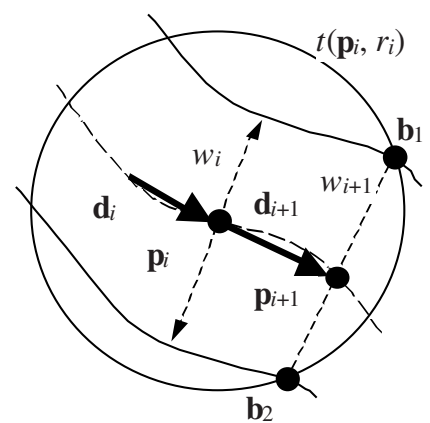

(b)

Fig. 3. Line elements of a gray scale image.

$$
\mathbf{p}_{i+1}=\frac{\mathbf{b}_{1}+\mathbf{b}_{2}}{2} \quad \mathbf{d}_{i+1}=\mathbf{p}_{i+1}-\mathbf{p}_{i} \quad w_{i+1}=\left\|\mathbf{b}_{1}-\mathbf{b}_{2}\right\|
$$

The border points $\mathbf{b}_{1}$ and $\mathbf{b}_{2}$ are detected by analyzing the gray level intensity of the circular pattern $t$. The circular pattern is a set of $\lfloor 2 \pi r\rfloor$ samples taken over the perimeter of the circumference with center $\mathbf{p}_{i}$ and radius $r$, in other words, $t(\mathbf{p}, r)=\left(c_{0}\right.$, $\left.c_{1}, \ldots, c\lfloor 2 \pi r\rfloor\right)$, where 


$$
c_{i}=I(p x+|\mathbf{p}| \cos (i \delta \theta), p y+|\mathbf{p}| \operatorname{sen}(i \delta \theta))
$$

for $i=1,2, \ldots,\lfloor 2 \pi r\rfloor-1$ and $\delta \theta=2 \pi\lfloor 2 \pi r\rfloor$. Due to discrete nature of the gray scale image $I$, the samples $c i$ are computed using bilinear interpolation. Then, the first derivative of $t(\mathbf{p}, r), d t(\mathbf{p}, r)$, is computed using finite differences. The local maximum of the magnitude $|d t(\mathbf{p}, r)|$ is detected and grouped in to pairs by means of the sign in $d t(\mathbf{p}, r)$. Given a pair of border points, it is reported a line element only if the magnitude of $|d t(\mathbf{p}, r)|$ in the border points is larger than a threshold. On the opposite way, it begins a new scan procedure. In our practical implementation, we use only one half of the circular pattern, as Fig. 4(a) shows in a zoomed detail. The gray level of the pattern is shown in Fig. 4(b). The Fig. 5(a) shows the whole spine extraction of a stripe image and the Fig. 5(b) presents a detail.

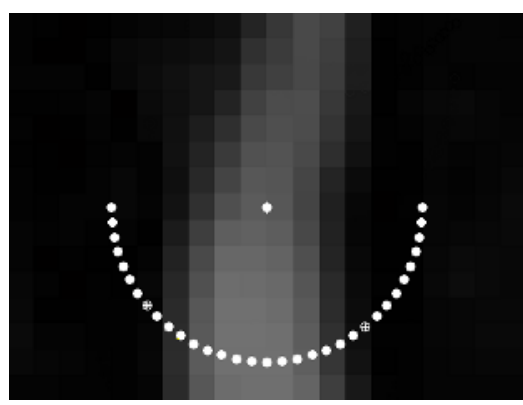

(a)

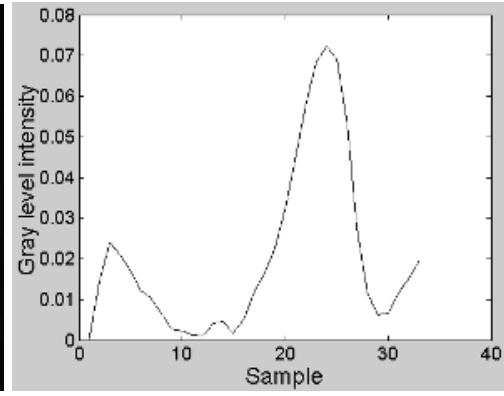

(b)

Fig. 4. Circular pattern

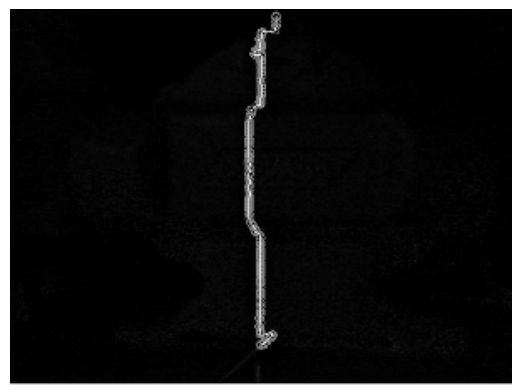

(a)

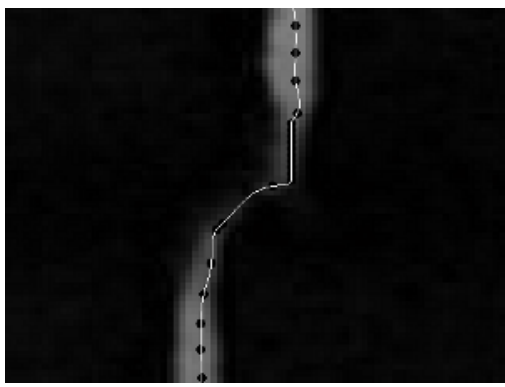

(b)

Fig. 5. Spine extraction with subpixel accuracy.

\section{Hardware and Software Platform}

The layout of the computer vision system for dimensional measurement using computer vision is presented in Fig. 6(a). In this figure, a PC equipped with a DT3155 
frame grabber [3] acquires 640X480X8 planar images incoming from a high resolution CCD camera. In order to achieve real time process, the algorithm described in the previous section was written in Visual $\mathrm{C}++$ 6.0. The user interface is shown in Fig. 6(b).

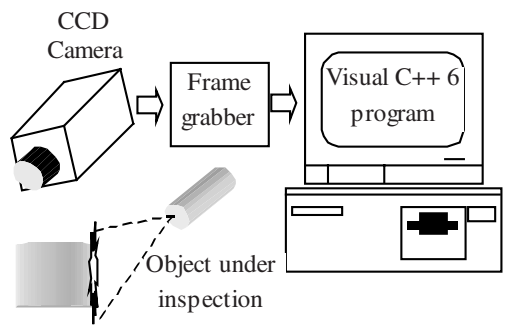

(a)

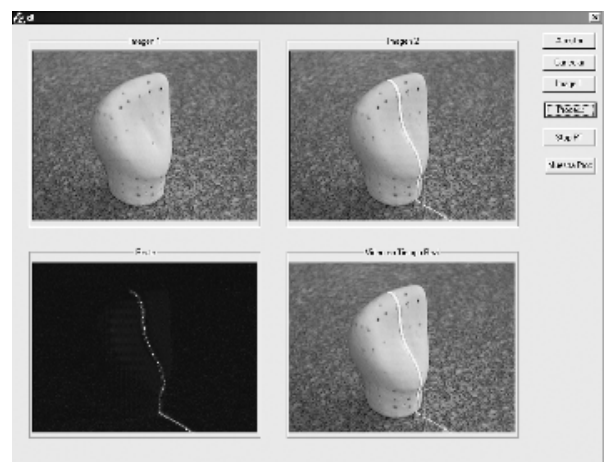

(b)

Fig. 6. Hardware and software platform for dimensional measurement using computer vision.

\section{Experimental Results}

Some experimental results can be reported with the 3D reconstruction of the spine extracted from the stripe image. The experimental arrangement, Fig. 7, is supported by a coordinate measuring machine with the purpose of system calibration. After laser plane and camera calibration, the reconstruction is implemented with a similar approach as described in [5]. In our test setup, the object under inspection is a scaled model of a tooth. It is easy to prove that the reconstruction problem can be stated as follows.

$$
\left[\begin{array}{ccc}
r_{7} \frac{X_{u}}{f}-r_{1} & r_{8} \frac{X_{u}}{f}-r_{2} & r_{9} \frac{X_{u}}{f}-r_{3} \\
r_{7} \frac{Y_{u}}{f}-r_{4} & r_{8} \frac{Y_{u}}{f}-r_{5} & r_{9} \frac{Y_{u}}{f}-r_{6} \\
A & B & C
\end{array}\right]\left[\begin{array}{c}
T_{x}-T_{z} \frac{X_{u}}{f} \\
T_{y}-T_{z} \frac{Y_{u}}{f} \\
-D
\end{array}\right]=\left[\begin{array}{c}
x_{w} \\
y_{w} \\
z_{w}
\end{array}\right]
$$

and

$$
\begin{aligned}
& X_{d}=\frac{d p_{x}}{s x}\left(X_{f}-c_{x}\right) \quad Y_{d}=d p_{y}\left(Y_{f}-c_{y}\right) \\
& X_{u}=X_{d}\left[1+k\left(X_{d}^{2}+Y_{d}^{2}\right)\right\rfloor \quad Y_{u}=Y_{d}\left[1+k\left(X_{d}^{2}+Y_{d}^{2}\right)\right\rfloor
\end{aligned}
$$

where $\left\{r_{i}, T_{x}, T_{y}, T_{z}, f, d p_{x}, d p_{y}, k, c_{x}, c_{y}\right\}$ are camera calibration parameters, $\{A, B, C$, $D\}$ are laser plane calibration parameters, $\left(X_{f}, Y_{f}\right)$ are coordinates in the digitized image, $\left(X_{d}, Y_{d}\right)$ are image distorted coordinates, $\left(X_{u}, Y_{u}\right)$ are image undistorted 
coordinates and $\left(x_{w}, y_{w}, z_{w}\right)$ are the 3D reconstructed coordinates of the 2D feature $\left(X_{f}\right.$, $\left.Y_{f}\right)$. Then, using simple matrix algebra, the 3D coordinates can be computed from equation (1). Applying equation (1) to the extracted spine, the 3D plot of Fig. 8 is obtained. Comparing the measurement outcomes using the computer vision system described here against the one performed with coordinate measuring machine, we compute an RMS reconstruction error of $0.08 \mathrm{~mm}$ in a $100 \times 100 \times 100 \mathrm{~mm}$ measurement volume.

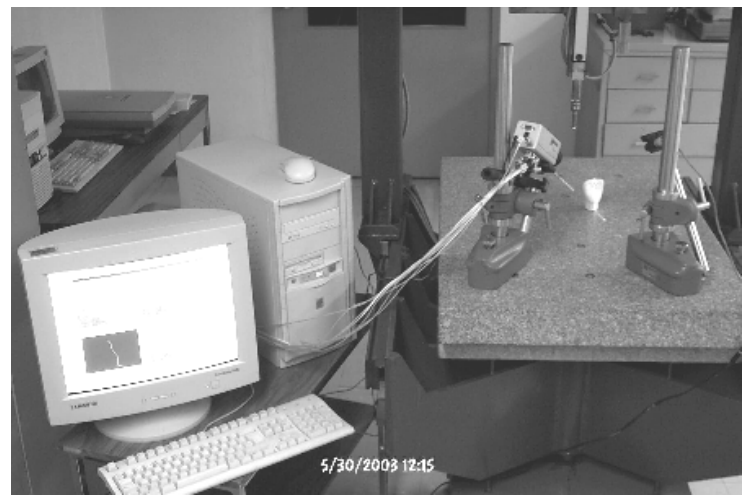

Fig. 7. Experimental setup.

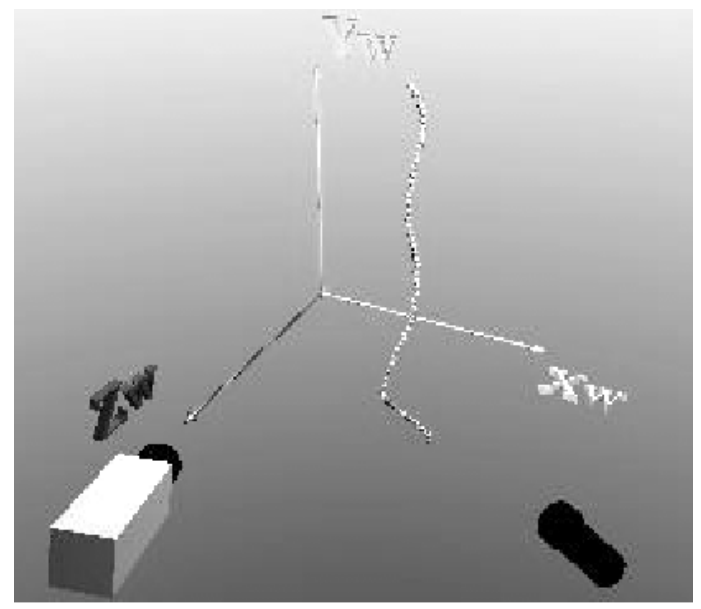

Fig. 8. Plot of a 3D reconstruction.

\section{Conclusions and Future Work}

A vision system for subpixel laser profile extraction was presented. The system accomplishes with high accuracy in both, image processing and 3D reconstruction. An algorithm for feature extraction with real time operation was tested in an 
experimental setup. The geometric oriented approach of the image processing algorithm contributes to an easy implementation using Visual $\mathrm{C}++$. For this reason we discard the use of third party software for image or mathematical processing; our developed software was implemented using basic development tools. The results oriented our future work in the following directions.

1. The development of an electro-mechanical device in order to scan the whole geometry of 3D work pieces. In our concept, a rotary support with angular position feedback is needed to perform a complete measurement process.

2. The incorporation of $3 \mathrm{D}$ graphics in the user interface. We are planning the development of real time render in the user interface using, for example, OpenGL.

3. The calibration improvement. We are planning to build a rotary support and calibrate it with the use of higher accuracy standards, like coordinate measuring and form scan machines.

Acknowledgments. This work has been financially supported by DGAPA-UNAM. The authors of this paper are indebt to the grant No IN111001 from PAPIIT.

\section{References}

1. Aguilar, J., Torres, F., Lope, M.A.: Stereo Vision for 3D Measurement: Accuracy Analysis, Calibration and Industrial Applications. Measurement 184 (1996) 193-200.

2. Chi, L.-C., Chih, L.-Y.: A new approach to high precision 3-D measuring system. Image and Vision Computing 17 (1999) 805-814.

3. Data Translation: SDK DT-Active Open Layers User's Manual. Data Translation, USA (1999).

4. Harris, K., Efstratiadis, S.N., Maglaveras, N., Gourassas, J., Pappas, C., Louridas, G.: Coronary Arterial Tree Extraction based on Artery Tracking and Mathematical Morphology. Computers in Cardiology 25 (1998) 769-772.

5. Izquierdo, M.A.G., Sánchez, M.T., Ibáñez, A., Ullate, L.G.: Sub-pixel measurement of 3D surfaces by laser scanning. Sensors and Actuators 76 (1999) 1-8.

6. Steger, C.: Subpixel-Precise Extraction of Lines and Edges. International Archives of Photogrammetry and Remote Sensing XXXIII (2000) 141-156.

7. Tsai, R.Y.: A Versatile Camera Calibration Technique for High-Accuracy 3D Machine Vision Metrology Using Off-the-Shelf TV Cameras and Lenses. IEEE Journal of Robotics and Automation 34 (1987) 323-344.

8. Weng, J., Cohen, P. and Herniou, M.: Camera Calibration with Distortion Models and Accuracy Evaluation. IEEE Transactions on Pattern Analysis and Machine Intelligence 1410 (1992) 965-980. 